\title{
Patient-controlled analgesia after shoulder surgery
}

\begin{abstract}
The shoulder joint has some specific properties in the human body. Shoulder surgery is often associated with severe postoperative pain, particularly within the first 48 hours. The management of acute postoperative pain after shoulder surgery has been performed using conventional nonsteroidal anti-inflammatory drugs (NSAIDs), local injection of analgesics, i.v. patient-controlled analgesia (PCA), continuous intrabursal infusion of analgesics, and patient-controlled interscalene or subacromial analgesia. When considering the effectiveness of pain control, the incidence of side effects, and patient satisfaction, patientcontrolled interscalene analgesia may be superior.
\end{abstract}

Keywords: Shoulder surgery, Patient-controlled analgesia, Interscalene
Volume I Issue 3 - 2014

\author{
Ahmet Eroglu \\ Anesthesiology and Intensive Care Medicine, Karadeniz \\ Technical University, Turkey
}

Correspondence: Ahmet Eroglu, Anesthesiology and Intensive Care Medicine, Karadeniz Technical University, 61000, Trabzon, Turkey, Tel 90-462-377-5900, Email erogluah@hotmail.com

Received: July 04, 2014 | Published: July 10, 2014
Abbreviations: NSAIDS, Non-Steroidal Anti-Inflammatory Drugs; PCA, Patient-Controlled Analgesia; PCIA, Patient - Controlled Interscalene Analgesia

\section{Introduction}

The shoulder joint has some specific properties in the human body. It has adjusted to the mechanical demands of a vertical body position more than any other joint. The shoulder has the largest motion range of all joints in the human body. In addition to the shoulder is exposed cosmetically, particularly in women. Extensive surgical exposures of the shoulder joint were commonly used when shoulder surgery first began. With the advent of arthroscopy, shoulder surgery has become even less invasive. The principles of surgical procedures to the shoulder, however, have largely remained unchanged. Shoulder surgery is often associated with severe postoperative pain, particularly within the first 48 hours. Postoperative pain after shoulder surgery can cause patient discomfort as well as compromise functional recovery. Adequate pain relief after shoulder surgery is necessary both for the comfort of the patients and for an early use of rehabilitation exercise. The management of acute postoperative pain after shoulder surgery has been performed using conventional nonsteroidal anti-inflammatory drugs (NSAIDs), local injection of analgesics, i.v. patient-controlled analgesia (PCA), continuous intrabursal infusion of analgesics, and patient-controlled interscalene or subacromial analgesia. ${ }^{1-10}$

\section{Regional anesthesia techniques and clinical studies}

Regional anesthesia techniques provide important advantages compared with general anesthesia in orthopedic surgeries. Regional anesthesia is one of the important methods for multimodal postoperative pain control. Regional anesthesia would provide excellent pain control and improve outcome such as decrease in side effects, improvement of pulmonary function, prevention of chronic pain, or reduction in hospital stay. Thus the regional anesthetic techniques and outcome using regional anesthesia for postoperative pain have becoming one of the important fields.

\section{Patient-Controlled Analgesia (PCA)}

Patient-controlled analgesia is considered a measurement standard for assessing acute postoperative pain treatment. This technique has proved to be popular because patients like the security of knowing they can achieve pain relief quickly and easily without involving a nurse. However, potent opioids result in several potential side effects, including nausea, pruritus, urinary retention, and respiratory depression. Interscalene brachial plexus block is often used to provide anesthesia and analgesia for shoulder surgery. ${ }^{1}$ When prolonging the block with a patient-controlled interscalene analgesia (PCIA) infusion $0.15 \%$ bupivacaine or ropivacaine provide adequate pain relief, and high patient satisfaction after shoulder surgery. ${ }^{2}$ The use of PCIA both with $0.15 \%$ bupivacaine and $0.2 \%$ ropivacaine were demonstrated to provide better quality of pain control, decreased incidence of side effects such as vomiting and pruritus than PCA with opioids after major shoulder surgery. ${ }^{3,4}$

Borgeat et al. ${ }^{5}$ reported that PCIA with $0.2 \%$ ropivacaine and $0.15 \%$ bupivacaine provided a similar pain relief after major shoulder surgery. However, ropivacaine $0.2 \%$ was associated with better preservation of strength in the hand and less paresthesia in the fingers. Casati et al. ${ }^{1}$ reported that PCIA with $0.125 \%$ levobupivacaine provided adequate pain relief after major open shoulder surgery, with a reduced volume of local anesthetic infused during the first postoperative day and no differences in the recovery of motor function as compared with $0.2 \%$ ropivacaine. Opioids and local anesthetics given into subacromial bursa have been used for pain relief after shoulder surgery. The continuous subacromial infusion of these analgesics for postoperative analgesia is controversial. Boss et $\mathrm{al}^{6}{ }^{6}$ reported that bupivacaine $0.25 \%$ at a rate of $6 \mathrm{~mL} / \mathrm{h}$ given as infusion in the subacromial space was ineffective for postoperative pain control in open acromioplasty. Harvey et al. ${ }^{7}$ reported that the use of subacromial ropivacaine $0.25 \%$ as PCA provided effective control of postoperative pain.

Eroglu ${ }^{8}$ reported that the PCA techniques using subacromial ropivacaine or fentany i.v. provided similar and adequate pain relief and minimal side-effects after open acromioplasty surgery. But, the PCA using subacromial fentanyl was not as effective as either subacromial ropivacaine or i.v. fentanyl. More than $40 \%$ of discharged ambulatory patients undergoing orthopedic procedures suffer from moderate-to-severe postoperative pain at home. It has demonstrated that ropivacaine or bupivacaine infusion using a portable infusion pump and an interscalene perineural catheter at home decreased pain, narcotic used and related side effects, sleep disturbances, and improved patient satisfaction. ${ }^{9,10}$

\section{Conclusion}

Patient-controlled postoperative analgesia using interscalene, subacromial, and intravenous catheter can provide pain relief after shoulder surgery. When considering the effectiveness of pain control, 
the incidence of side effects, and patient satisfaction, patient-controlled interscalene analgesia may be superior.

\section{Funding details}

None.

\section{Acknowledgments}

None.

\section{Conflicts of interests}

Authors declare that there is no conflict of interest.

\section{References}

1. Casati A, Borghi B, Fanelli G, et al. Interscalene brachial plexus anesthesia and analgesia for open shoulder surgery:a randomized, double-blinded comparison between levobupivcaine and ropivacaine. Anesth Analg. 2003;96(1):253-259.

2. Eroglu A, Uzunlar H, Sener M, et al. A clinical comparison of equal concentration and volume of ropivacaine and bupivacaine for interscalene brachial plexus anesthesia and analgesia in shoulder surgery. Reg Anesth Pain Med. 2004;29(6):539-543.

3. Borgeat A, Schappi B, Biasca N, et al. Patient-controlled analgesia after major shoulder surgery:patient-controlled interscalene analgesia versus patient-controlled analgesia. Anesthesiology. 1997;87(6):1343-1347.
4. Borgeat A, Tewes E, Biasca N, et al. Patient-controlled interscalene analgesia with ropivacaine after major shoulder surgery:PCIA vs PCA. Br J Anaesth. 1998;81(4):603-605.

5. Borgeat A, Kalberer F, Jacob H, et al. Patient-controlled interscalene analgesia with ropivacaine $0.2 \%$ versus bupivacaine $0.15 \%$ after major open shoulder surgery:the effects on hand motor function. Anesth Analg. 2011;92(1):218-223.

6. Boss AP, Maurer T, Seiler S, et al. Continuous subacromial bupivacaine infusion for postoperative analgesia after open acromioplasty and rotator cuff repair :preliminary results. J Shoulder Elbow Surg. 2004;13(6):630-634.

7. Harvey GP, Chelly JE, AlSamsam T, et al. Patient-controlled ropivacaine after arthroscopic subacromial decompression. Arthroscopy. 2004;20(5):451-455.

8. Eroglu A. A comparison of patient-controlled subacromial and i.v. analgesia after open acromioplasty surgery. $\mathrm{Br} J$ Anaesth. 2006;96(4):497-501.

9. Rawal N, Allvin R, Axelsson K, et al. Patient-controlled regional analgesia (PCRA) at home. controlled comparison between bupivacaine and ropivacaine brachial plexus analgesia. Anesthesiology. 2002;96(6):1290-1296.

10. Ilfeld BM, Morey TE, Wright TW, et al. Continuous interscalene brachial plexus block for postoperative pain control at home:a randomized, double-blinded, placebo-controlled study. Anesth Analg. 2003;96(4):1089-1095. 